\title{
SUSY searches in lepton and photon final states at the LHC
}

\author{
Jan-Frederik Schulte* \\ Rheinisch-Westfaelische Tech. Hoch. (DE) \\ now at Purdue University (US) \\ on behalf of the ATLAS and CMS collaborations \\ E-mail: jan-frederik.schultedcern.ch
}

The datasets recorded by the ATLAS and CMS collaborations in 2015, at the increased center-ofmass energy $\sqrt{s}=13 \mathrm{TeV}$, greatly increase the sensitivity of searches for Supersymmetry at the LHC. Leptons and photons can be reconstructed and identified with high precision and efficiency. Therefore, searches for signatures including these particles offer large reduction and good control of backgrounds, complementing the searches for fully hadronic signatures. Additionally, searches with $\tau$ leptons or photons are sensitive to different mechanisms of Supersymmetry breaking, such as gauge mediated supersymmetry breaking. Presented here are a variety of searches for leptonic signatures, differing by lepton flavours, multiplicity, and charge. Also, searches for diphoton signatures are shown. In most searches, no excess above expectation from Standard Model processes are observed and exclusion limits are set. However, a moderate excess is observed in a search for opposite-sign, same-flavour leptons performed by the ATLAS collaboration, using the same selection where a similar excess was already observed by ATLAS on the $\sqrt{s}=8 \mathrm{TeV}$ dataset. This excess is not present in the corresponding CMS search.

Fourth Annual Large Hadron Collider Physics

13-18 June 2016

Lund, Sweden

${ }^{*}$ Speaker. 


\section{Introduction}

The search for Supersymmetry (SUSY) is one of the main focal points of the physics programme at the CERN LHC [1]. A comprehensive set of searches is performed by both the ATLAS and CMS collaborations [2,3]. A large emphasis is put on searches for models in which the Rparity $P_{\mathrm{R}}=-1^{3 B+L+2 s}$, with $\mathrm{B}, \mathrm{L}$ and s denoting the baryon and lepton number and the spin of the particle, is a conserved quantity. Therefore, SUSY particles are pair produced and the lightest SUSY particle (LSP) is stable. R-Parity conservation is assumed in all searches presented here. In most MSSM-inspired models, the lightest neutralino $\tilde{\chi}_{1}^{0}$ is the LSP. However, in models with gauge-mediated SUSY breaking (GMSB), this role is assumed by the gravitino $\tilde{G}$.

Leptons and photons are reconstructed and identified with high efficiency and precision by both the ATLAS and CMS detectors. They can be used to reduce and control the large backgrounds from standard model (SM) processes. Also, the presence of $\tau$ leptons or photons can be favoured, for example in GMSB models, and can therefore greatly increase sensitivity to such models. Here, several searches performed by ATLAS and CMS using the dataset collected at a center-mass-energy $\sqrt{s}=13 \mathrm{TeV}$ in 2015 are presented. The dataset corresponds to $3.2 \mathrm{fb}^{-1}\left(2.3 \mathrm{fb}^{-1}\right)$ for ATLAS (CMS). The results are presented grouped by final state.

\section{Searches with two opposite-sign leptons}

The production of two opposite-sign (OS), same-flavor (SF) light leptons (e, $\mu$ ) can occur in the decay chains of the initially produced heavy SUSY particles either via the production of $\mathrm{Z}$ bosons or decays such as $\tilde{\chi}_{2}^{0} \rightarrow \tilde{\chi}_{1}^{0} \ell^{+} \ell^{-}$via an intermediate slepton $\tilde{l}$. In the first case, the signature will be an excess at the $\mathrm{Z}$ boson mass in the distribution of the dilepton invariant mass $m_{\ell \ell}$ (on-Z), while the latter case results in a triangular edge shape with an endpoint given by the mass difference of the neutralinos (off-Z). In the $\sqrt{s}=8 \mathrm{TeV}$ datasets, CMS observed an excess with a local significance of $2.6 \sigma$ for $m_{\ell \ell}$ below the $\mathrm{Z}$ boson mass, as illustrated on the left side of Figure 1. This excess was not confirmed by ATLAS in their corresponding analysis. However, ATLAS observed an excess with a local significance of $3.0 \sigma$ in their on- $Z$ search, as shown on the right side of Figure 1, in a signal region with high missing transverse energy $E_{\mathrm{T}}^{\text {miss }}$ and hadronic activity. CMS did not report a result in a corresponding region at $\sqrt{s}=8 \mathrm{TeV}$. Given this unclear picture, the results of the analysis of the $\sqrt{s}=13 \mathrm{TeV}$ datasets was highly anticipated. These results [6, 7] are summarized in the following.

In the on-Z search, targeting the excess observed by ATLAS at $\sqrt{s}=8 \mathrm{TeV}$, OS-SF lepton pairs with $81 \mathrm{GeV}<m_{\ell \ell}<101 \mathrm{GeV}$ are selected. Additionally, the events are required to have $E_{\mathrm{T}}^{\text {miss }}>$ $225 \mathrm{GeV}, \sum_{j e t s} p_{\mathrm{T}}+p_{\mathrm{T}}^{\ell_{1}, \ell_{2}}>600 \mathrm{GeV}$, and $\Delta \phi$ between $E_{\mathrm{T}}^{\text {miss }}$ and the two leading jets larger than 0.4 . CMS selects leptons with $p_{\mathrm{T}}>20 \mathrm{GeV}$, while in the ATLAS search the threshold is 50 (25) GeV for the leading (subleading) lepton. Backgrounds from SM processes to this selection are estimated with very similar methods in both CMS and ATLAS searches. Dominant are flavor-symmetric processes, such as top-pair or WW production. These processes produce the same amount of SF as $e \mu$ pairs. Therefore, their contribution to the signal region is estimated from $e \mu$ control samples, corrected for deviations from exact flavor-symmetry introduced by detector effects. Another source of background is $\mathrm{Z}+\mathrm{jets}$ production, where the large $E_{\mathrm{T}}^{\mathrm{miss}}$ is caused by jet mismeasurement. This 

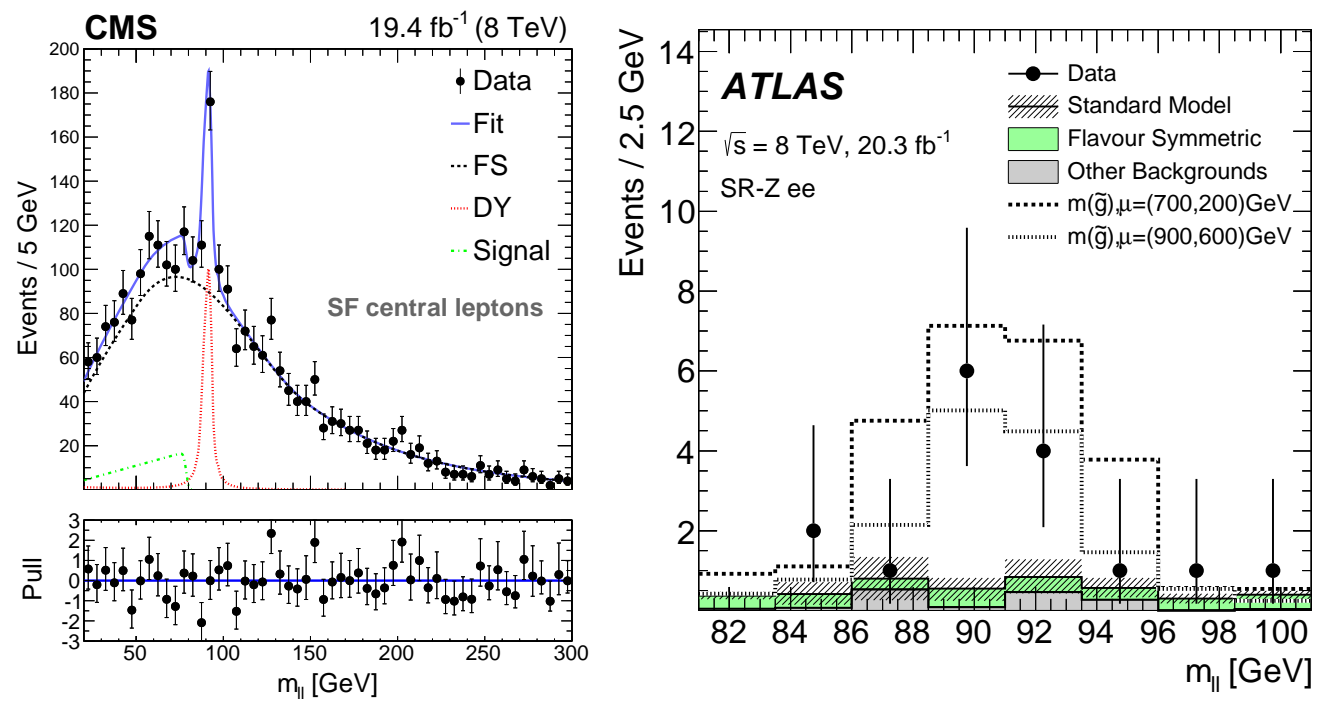

Figure 1: Left: Result of a fit to the $m_{\ell \ell}$ distribution in search for an kinematic edge in the CMS $\sqrt{s}=8 \mathrm{TeV}$ dataset [4]. Right: Result of the search for an excess in the $\mathrm{Z}$ boson mass window for dielectron events in the ATLAS $\sqrt{s}=8 \mathrm{TeV}$ dataset [5].

Table 1: Event yields in the CMS and ATLAS on-Z searches in the dataset recorded at $\sqrt{s}=13 \mathrm{TeV}$ in 2015.

\begin{tabular}{c|c|c} 
& Observed & Expected \\
\hline CMS & 14 & $12.3_{-2.8}^{+4.0}$ \\
ATLAS & 21 & $10.3 \pm 2.3$
\end{tabular}

contribution is estimated from $\gamma+$ jets events, which have the same signature of a well measured boson recoiling against hadronic jets. Backgrounds from more rare SM processes, such as WZ production, are estimated from simulation.

The results of the on-Z search are shown in Figure 2, where the left (right) side shows the CMS (ATLAS) result. No significant excess is observed in the CMS search, while ATLAS again observes as small excess, with a local significance of $2.2 \sigma$. The event yields are summarized in Table 1 . In addition to this signal region, targeting the excess observed by ATLAS at $\sqrt{s}=8 \mathrm{TeV}$, CMS has studied a variety of additional signal regions, which are not presented here. No significant excess has been observed in these regions. These results, together with the interpretation of all results in terms of simplified models for both ATLAS and CMS, can be found in the publications by the experiments.

Targeting the excess observed at $\sqrt{s}=8 \mathrm{TeV}$ for $m_{\ell \ell}$ below the $\mathrm{Z}$ boson mass, CMS has repeated their off-Z search. No ATLAS result for this signal selection is available. The signal selection has been kept the same as for the previous analysis, requiring $E_{\mathrm{T}}^{\text {miss }}>100 \mathrm{GeV} \& N_{\text {jet }} \geq 3$ or $E_{\mathrm{T}}^{\text {miss }}>150 \mathrm{GeV} \& N_{\text {jet }} \geq 2$, where $N_{\text {jet }}$ is the number of selected hadronic jets. Additionally, subregions either vetoing or requiring the presence of a b-tagged jet are defined. The $m_{\ell \ell}$ distribution is divided in five bins, three of which where present already in the analysis of the $\sqrt{s}=8 \mathrm{TeV}$ dataset, while the other close gaps in the acceptance between them. The event sample is further 

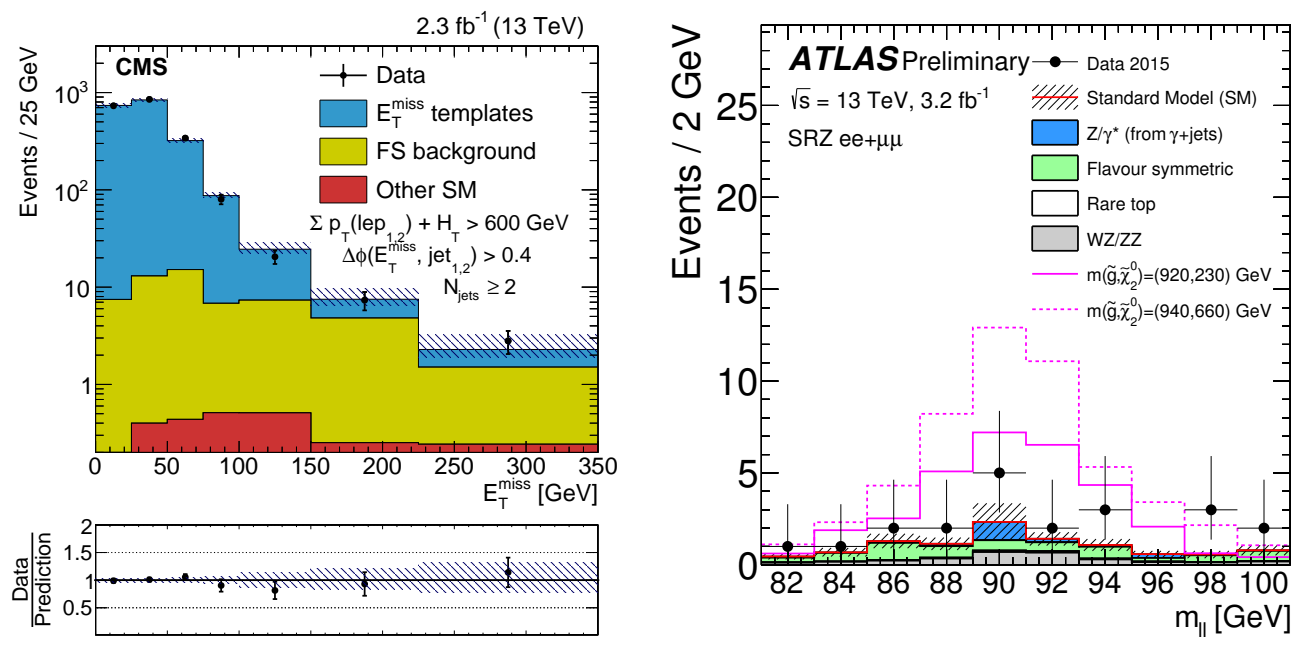

Figure 2: Left: $E_{\mathrm{T}}^{\text {miss }}$ distribution in the CMS on-Z search. The last bin shows the signal region. [6]. Right: Resulting $m_{\ell \ell}$ distribution in the ATLAS on-Z search [7].

subdivided by lepton pseudorapidity, requiring either both leptons to be reconstructed in the barrel region ("central") or at least one to be reconstructed in the endcaps of the CMS detector ("forward"). The SM backgrounds and their estimation are identical to the on-Z search. The left side of Figure 3 shows the $m_{\ell \ell}$ distribution in the signal region for central leptons. Good agreement is observed over the full mass range. The excess presented in the $\sqrt{s}=8 \mathrm{TeV}$ dataset has not been confirmed in the new data. The right side of Figure 3 summarizes the event yields in all signal regions of the off-Z search, including the b-tag bins. Good agreement is observed.
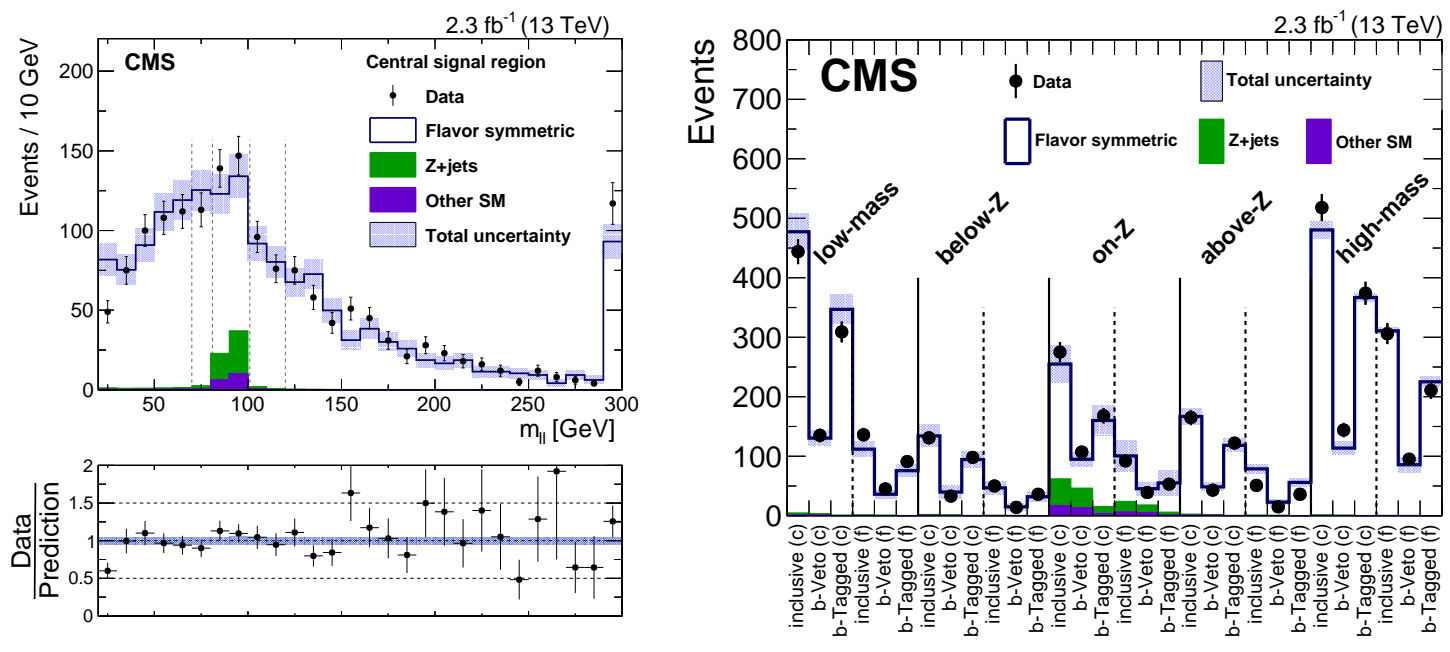

Figure 3: Left: Resulting $m_{\ell \ell}$ distribution in the CMS off-Z search for central leptons [6]. Right: Summary of event yields in all signal regions in the CMS off-Z search [6].

The results are interpreted in a simplified model of bottom-squark pair production, which features the production of OS-SF lepton pairs in the decay chains. The left side of Figure 4 shows the diagram for this model, while the right side shows the resulting exclusion limit in the $m_{\tilde{b}}-m_{\tilde{\chi}_{2}^{0}}$ plane. The limit reaches values of $m_{\tilde{b}}$ around $620 \mathrm{GeV}$ for low values of the neutralino mass. 

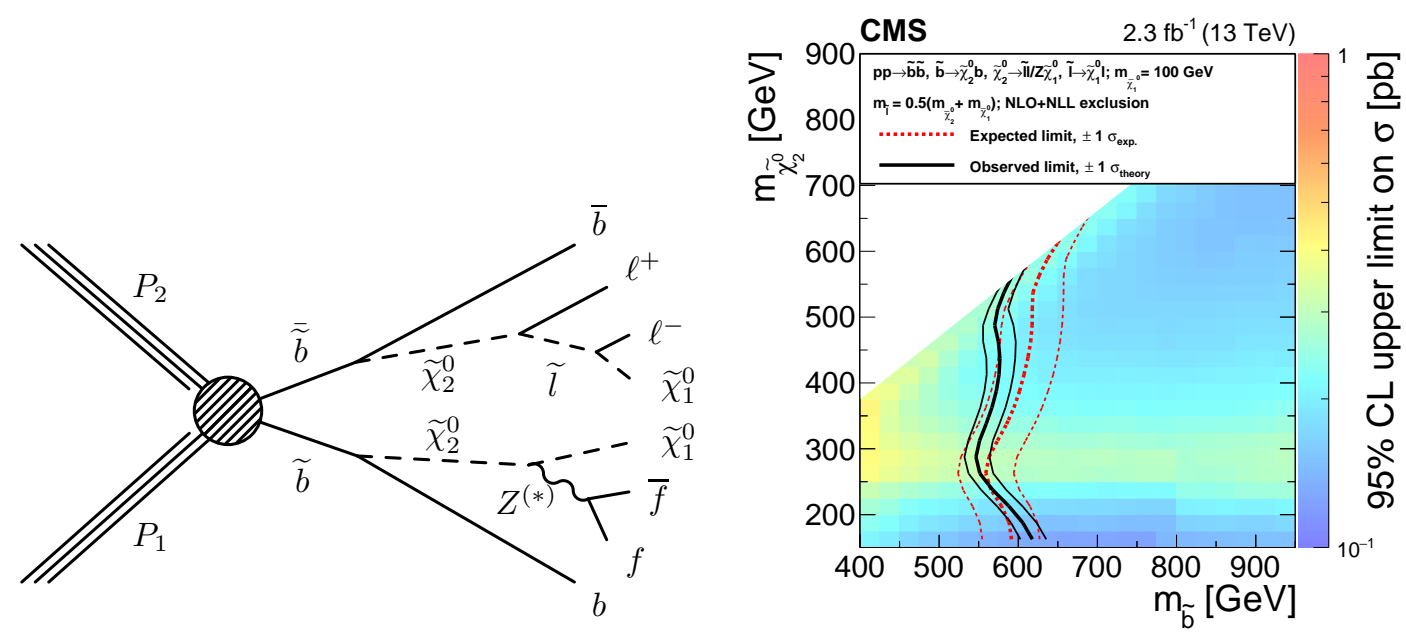

Figure 4: Left: Diagram for the simplified model used in the interpretation of the CMS off-Z search [6]. Right: Resulting exclusion limit in the $m_{\tilde{b}}-m_{\tilde{\chi}_{2}^{0}}$ plane [6].

\section{Searches with same-sign or multileptons}

Final states with two same-sign or multiple leptons are rare in the SM, but might be produced frequently in SUSY decay chains if they include sleptons, the production of SM vector bosons or multiple top quarks. Figure 5 illustrates four simplified models with these characteristics. Searches for these signatures have been performed by both the ATLAS and CMS collaborations $[8,9,10]$.
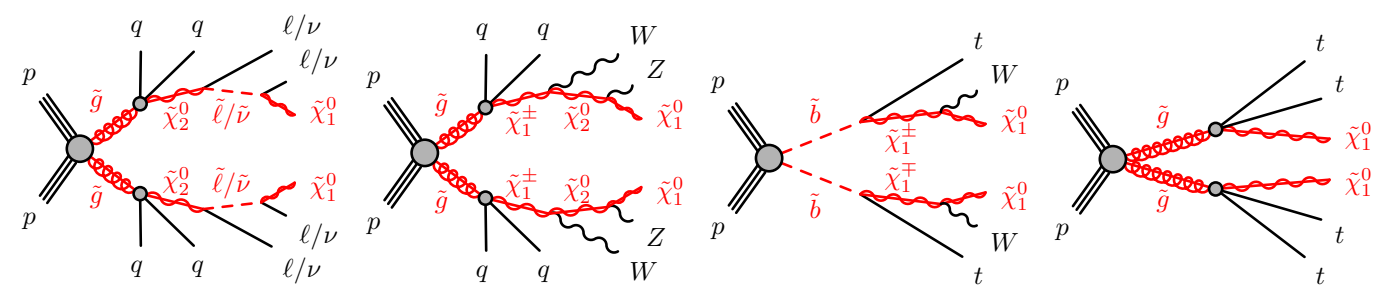

Figure 5: Diagrams of simplified models featuring the production of same-sign or multilepton final states [8].

There are three sources of SM backgrounds to these final states, which are common to all three analysis and estimated in similar ways: Non-prompt leptons, rare SM processes, and charge misidentification. In the first one, either jets are misidentified as leptons or the leptons originate from the decay of heavy-flavour mesons inside jets. Their contribution to the signal region is estimated from data control samples with relaxed isolation and identification criteria for the leptons. These events are then weighted by "tight-to-loose" ratios, also measured on data. The left side of Figure 6 shows the performance of such a method in the baseline selection of the CMS same-sign search. Several rare SM processes, such as diboson or $t \bar{t} V$ production, produce prompt samesign or multilepton events. These backgrounds are estimated from simulation, which has been either validated on or scaled to the data in control regions. The right side of Figure 6 shows the agreement between data and the simulation of WZ production in a validation region of the ATLAS 
search. Lastly, same-sign events can be produced by misidentifying the charge of a lepton in an OS pair. This background is estimated from OS control regions, multiplied with the probability of charge misidentification.
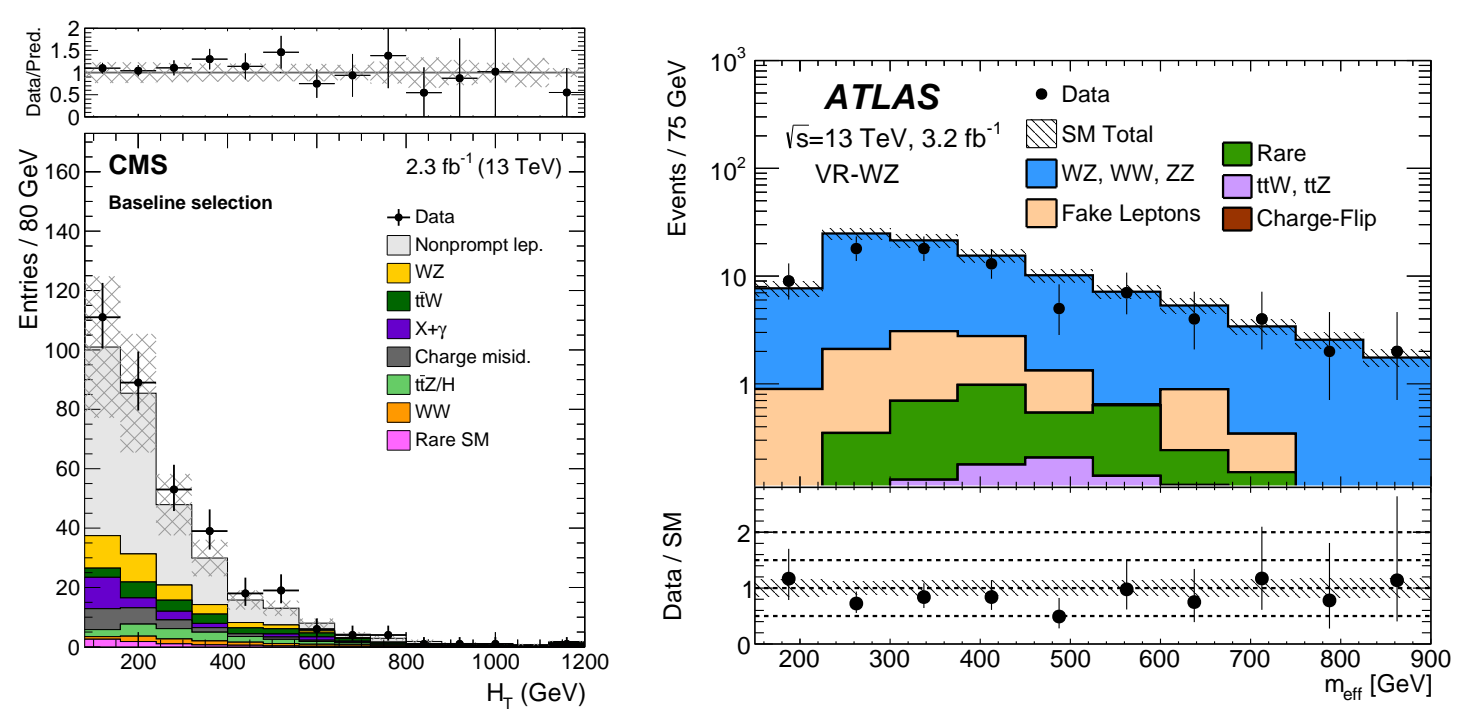

Figure 6: Left: Illustration of the performance of the estimation of non-prompt leptons from data in the CMS same-sign search [9]. Right: Data to simulation comparison in a verification region for the WZ background in the ATLAS multilepton search [8].

In a combined search for same-sign dilepton and multilepton events, ATLAS defines four search regions based on the number of leptons, jets, b-tagged jets, $E_{\mathrm{T}}^{\text {miss }}$, and the effective mass $m_{\text {eff }}$, defined as the scalar sum of the $p_{\mathrm{T}}$ of all selected objects and the $E_{\mathrm{T}}^{\text {miss }}$. These four regions target different regions of the signal phase space. Figure 7 shows the $E_{\mathrm{T}}^{\text {miss }}$ distribution in all four selections, without the $E_{\mathrm{T}}^{\text {miss }}$ requirement applied. Each time, the last bin corresponds to the signal region. No significant excess above the SM expectation is observed.

The results are interpreted in different simplified models. As an example, Figure 8 shows exclusion limits in the $m_{\tilde{g}}-m_{\tilde{\chi}_{1}^{0}}$ plane for the model shown in Figure 5 on the very left. The on $m_{\tilde{g}}$ limits reach $1.3 \mathrm{TeV}$ for light $\tilde{\chi}_{1}^{0}$. In general, mass limits reach $m_{\tilde{g}}$ of $1.1-1.3 \mathrm{TeV}$ and $m_{\tilde{\chi}_{1}^{0}}<550$ $850 \mathrm{GeV}$ in models of $\tilde{g}$ pair production. For models with $\tilde{b}$ pair production, the limits reach $m_{\tilde{b}}<540 \mathrm{GeV}$ for light $\tilde{\chi}_{1}^{0}$.

CMS has performed two distinct searches for same-sign and multilepton signatures. The samesign analysis targets six different simplified models, which motivates the study of a large variety of signal selections. The events are categorized by lepton $p_{\mathrm{T}}$, the minimal transverse mass $m_{\mathrm{T}}^{\min }, E_{\tilde{T}}^{\operatorname{miss}}$, the scalar sum of jet $p_{\mathrm{T}}\left(H_{\mathrm{T}}\right)$, and the number of jets and b-tagged jets. The total number of signal regions is 66. No significant deviation from SM expectation is observed, as shown for example on the left side of Figure 9 for the signal regions with lepton $p_{\mathrm{T}}>25 \mathrm{GeV}$. The results are interpreted in a multitude of simplified models. As an example, the right side of Figure 9 shows the exclusion limits for a model of $\tilde{b}$ pair production, where the $\tilde{b}$ decays according to $\tilde{b} \rightarrow t \chi_{1}^{-} \rightarrow t W^{-} \tilde{\chi}_{1}^{0}$. The limit on $m_{\tilde{b}}$ reaches $680 \mathrm{GeV}$ for light $\tilde{\chi}_{1}^{0}$. As for the ATLAS search, the limit on $m_{\tilde{g}}$ reaches up to $1.3 \mathrm{TeV}$ in models of $\tilde{g}$ pair production, depending on model parameters. 

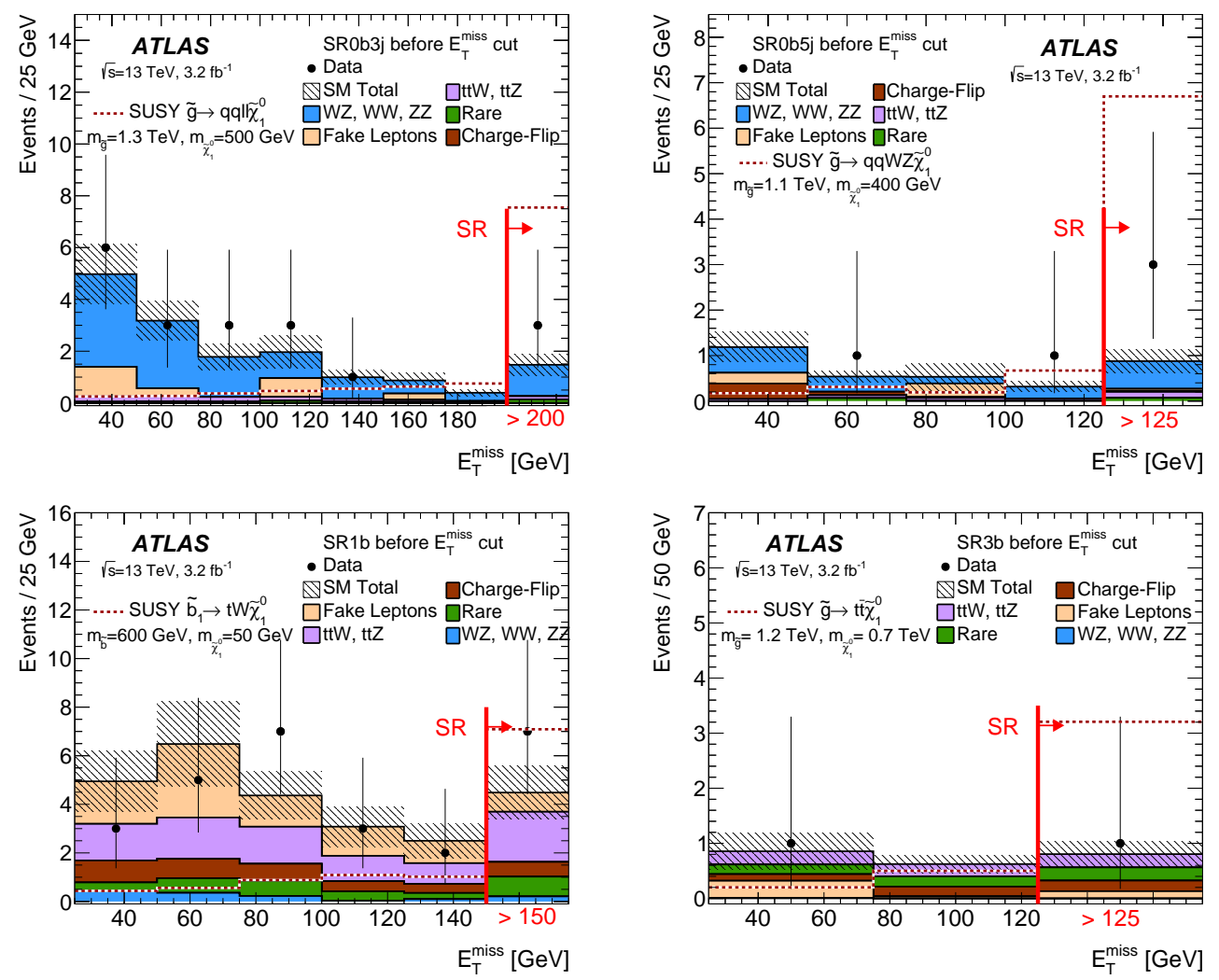

Figure 7: $E_{\mathrm{T}}^{\text {miss }}$ distribution in the four signal regions of the ATLAS same-sign and multilepton search [8].

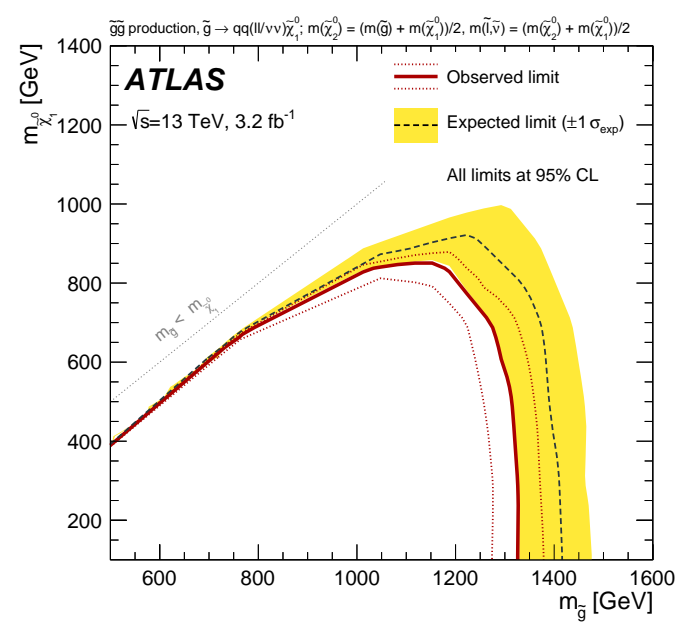

Figure 8: Exclusion limits in the $m_{\tilde{g}}-m_{\tilde{\chi}_{1}^{0}}$-plane for a simplified model of $\tilde{g}$ pair production in the ATLAS same-sign and multilepton search [8]. 

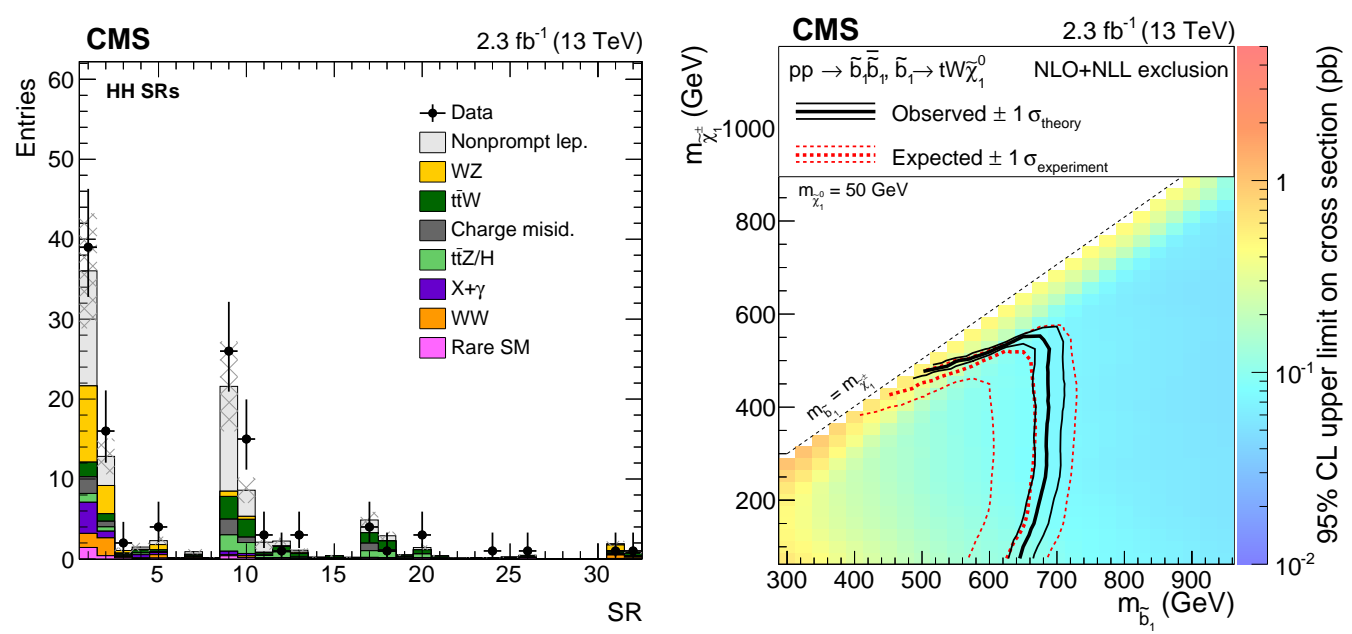

Figure 9: Left: Event yields in the CMS same-sign search for event categories with lepton $p_{\mathrm{T}}>25 \mathrm{GeV}$ [9]. Right: Exclusion limits in the $m_{\tilde{b}}-m_{\tilde{\chi}_{1}^{0}}$-plane for a model of $\tilde{b}$ pair production [9].

As the same-sign search, the CMS multilepton search targets a variety of simplified models in a variety of signal regions. The event sample is first divided by the presence of Z-boson candidate. The events are then further categorized based on the number of jets and b-tagged jets, $E_{\mathrm{T}}^{\mathrm{miss}}$, and $H_{\mathrm{T}}$. The event yields in all signal regions, compared to the expectation from SM backgrounds, are shown in Figure 10. No significant excess is observed.
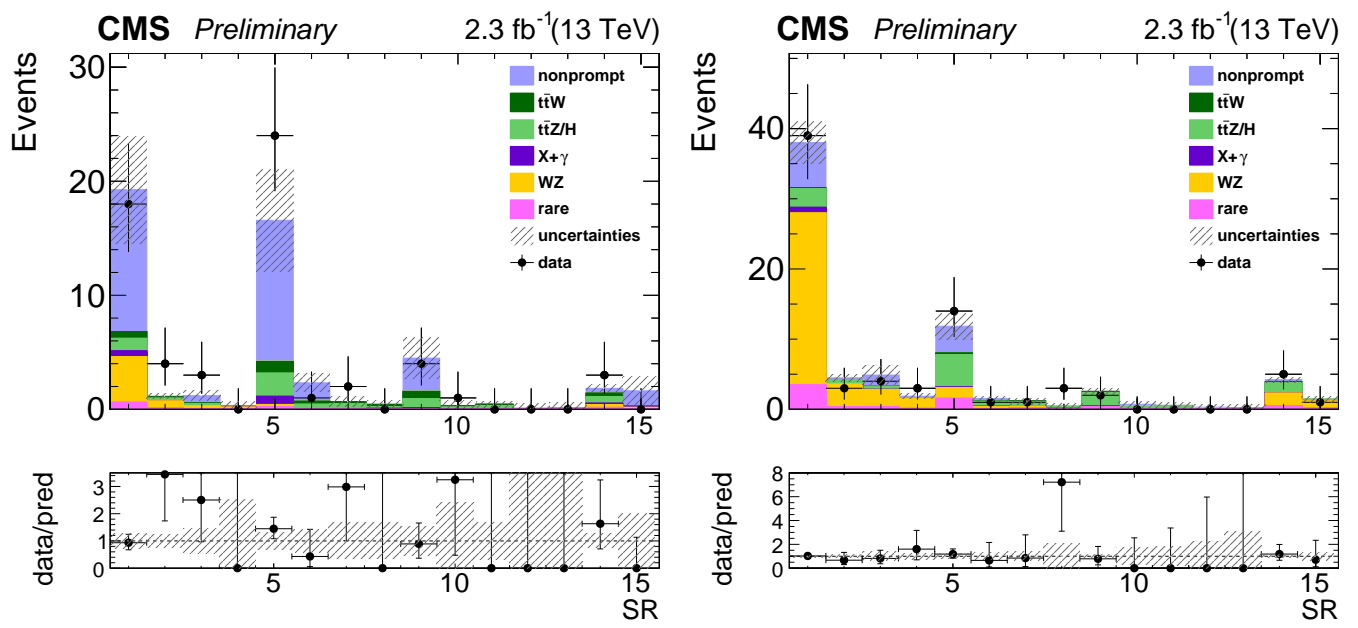

Figure 10: Event yields in all signal regions of the CMS multilepton search. The signal regions without (with) a Z-boson candidate are shown on the left (right) side [10].

The results are interpreted in several simplified models. Figure 11 shows as an example the exclusion limits in the $m_{\tilde{g}}-m_{\tilde{\chi}_{1}^{0}}$-plane for a model of $\tilde{g}$ pair production. The $\tilde{g}$ decays according to $\tilde{g} \rightarrow q \bar{q} \tilde{\chi}_{2}^{0} \rightarrow q \bar{q} Z \tilde{\chi}_{1}^{0}$ or $\tilde{g} \rightarrow q \bar{q}^{\prime} \tilde{\chi}_{1}^{ \pm} \rightarrow q \bar{q}^{\prime} W^{ \pm} \tilde{\chi}_{1}^{0}$. In this model, the limit on $m_{\tilde{g}}$ reaches $850 \mathrm{GeV}$, while for other models of $\tilde{g}$ pair production, limits reach $m_{\tilde{g}}$ of $1125 \mathrm{GeV}$ and $m_{\tilde{\chi}_{1}^{0}}$ of $650 \mathrm{GeV}$, depending on model parameters. For $m_{\tilde{\chi}_{0}^{1}}=200 \mathrm{GeV}, m_{\tilde{b}}<550 \mathrm{GeV}$ is excluded. 


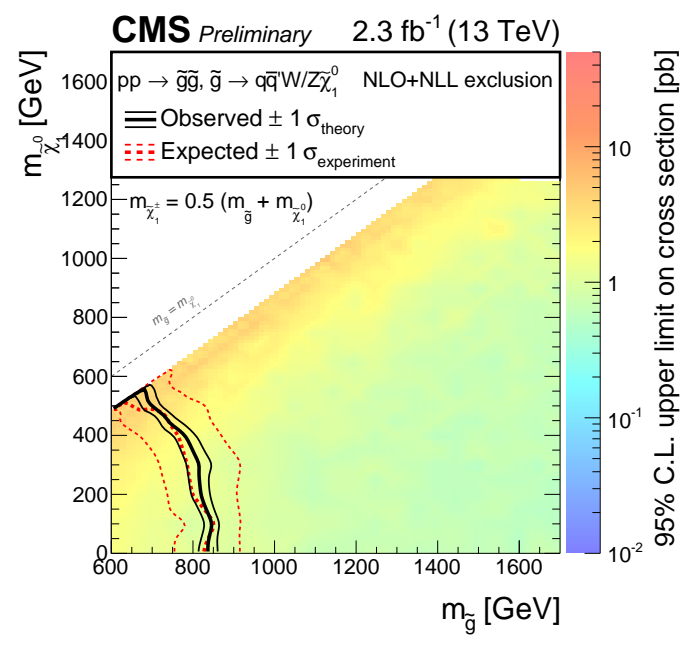

Figure 11: Exclusion limits in the $m_{\tilde{g}}-m_{\tilde{\chi}_{1}^{0}}$ plane in the CMS multilepton search for a simplified model of $\tilde{g}$ pair production [10].

\section{Search with $\tau$ leptons}

The ATLAS collaboration has searched for SUSY in final states with a single or multiple $\tau$ leptons [11]. This search is motivated by two distinct models. The first one is based on GMSB models where the $\tilde{\tau}_{1}$ is the next-to-lightest SUSY particle (NLSP) or co-NLSP together with $\tilde{e}_{R}$ and $\tilde{\mu}_{R}$. This leads to signatures as shown on the left side of Figure 12, where $\tilde{q}$ pair production is followed by cascade decays featuring the decay $\tilde{\chi}_{1}^{0} \rightarrow \tau \tau \tilde{G}$ via an intermediate $\tilde{\tau}$. The free parameters of this model are $\tan \beta$ and the SUSY breaking scale $\Lambda$. The second model is inspired by more generic models with light $\tilde{\tau}$ and features $\tilde{g}$ pair production, as shown on the right side of Figure 12.

The analysis is divided in two main channels: Exactly one $\tau$ and a veto on the presence of light leptons and two $\tau$ or more, allowing for the presence of light leptons. In each case, three signal regions are defined using $E_{\mathrm{T}}^{\text {miss }}, m_{\mathrm{T}}^{\tau}, H_{\mathrm{T}}, m_{\mathrm{eff}}$, and $m_{\mathrm{T} 2}^{\tau \tau}$. These regions target different mass ranges of the SUSY particles. Figure 13 shows the good agreement between data and simulation after a basic preselection in the both the single $\tau$ and the $\geq 2 \tau$ channels.

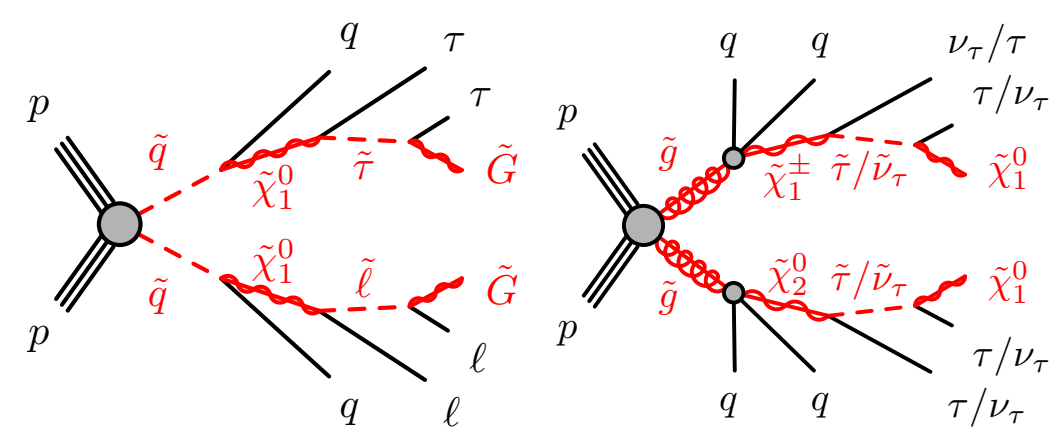

Figure 12: Left: Diagram for the model based on GMSB in the ATLAS $\tau$ lepton search [11]. Right: Diagram for the $\tilde{g}$ pair production model in the ATLAS $\tau$ lepton search [11]. 

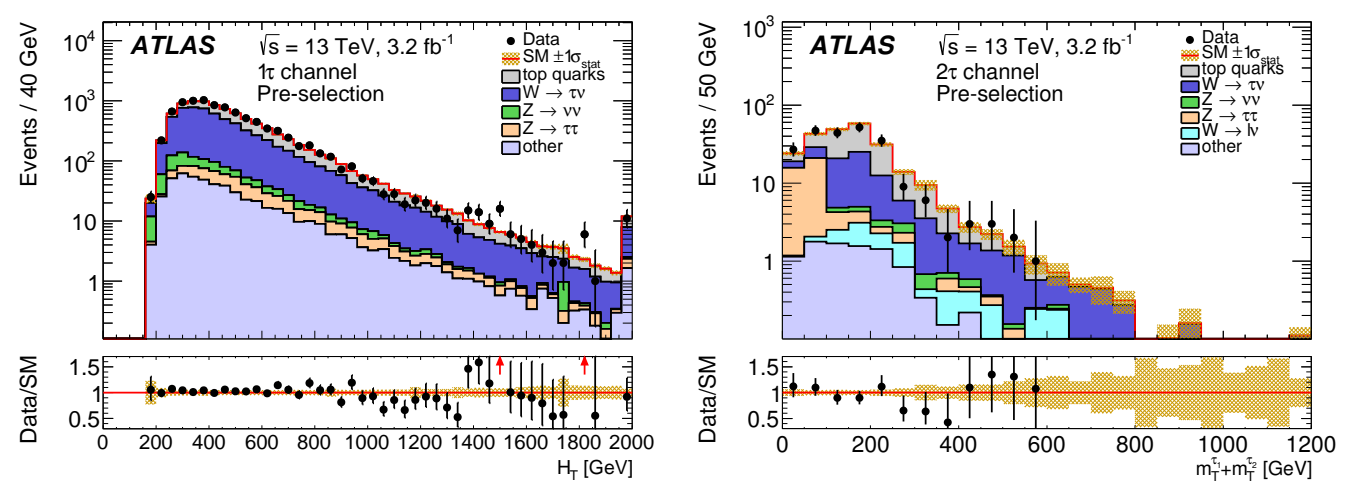

Figure 13: Left: $H_{\mathrm{T}}$ distribution after preselection for single $\tau$ in the ATLAS $\tau$ lepton search [11]. Right: Distribution of the sum of $m_{\mathrm{T}}^{\tau}$ for both $\tau$ after preselection for $\geq 2 \tau$ in the ATLAS $\tau$ lepton search [11].

The dominant SM backgrounds to this search are vector boson and top-pair production. The shapes of the background distributions are taken form simulation, while the normalizations are determined in a simultaneous fit to data in several control regions. A subdominant source of background are QCD events where jets are misreconstructed as hadronically decaying $\tau$. These are estimated from data using a jet-smearing technique in the single $\tau$ channel and a "tight-to-loose" ratio method for the $\geq 2 \tau$ channel. The performance of these methods is tested by comparing the observed data yields to the background estimation in several validation regions. These regions are designed to enhance the contribution of different background processes. The result of the validation is summarized in Figure 14. There is good agreement between the observed data and the background estimation after normalizing the backgrounds to the result of the simultaneous fit.

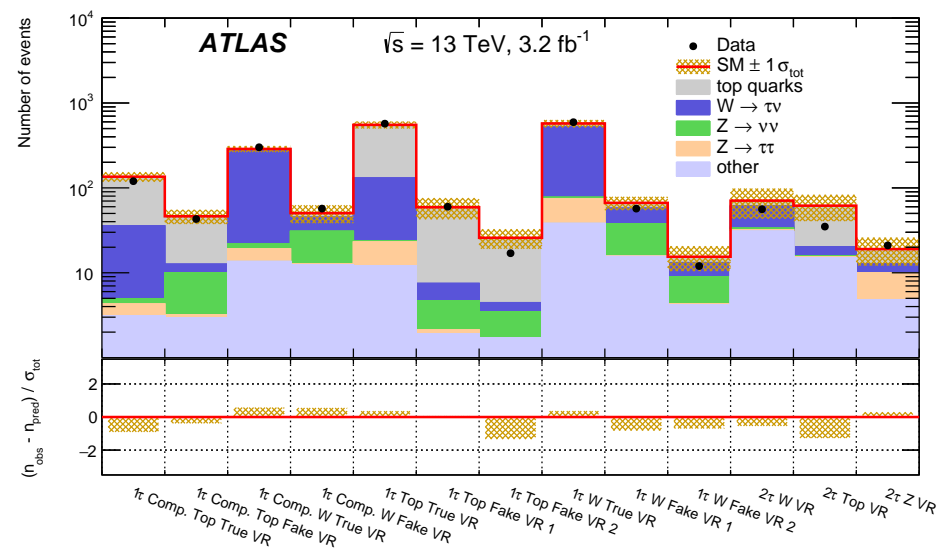

Figure 14: Validation of the estimation of SM backgrounds in several validation regions [11].

In all six signal regions, good agreement between the observed data and the background estimates is observed. As an example, Figure 15 shows the final $m_{\mathrm{T}}^{\tau}$ distributions for the three signal regions in the single $\tau$ search. The black arrows indicate the minimal value of $m_{\mathrm{T}}^{\tau}$ used in the signal region definition.

Exclusion limits are set for both considered models. The result for the $\tilde{g}$-pair production model is shown on the left side of Figure 16. Comparing the grey area to the observed exclusion curve, 

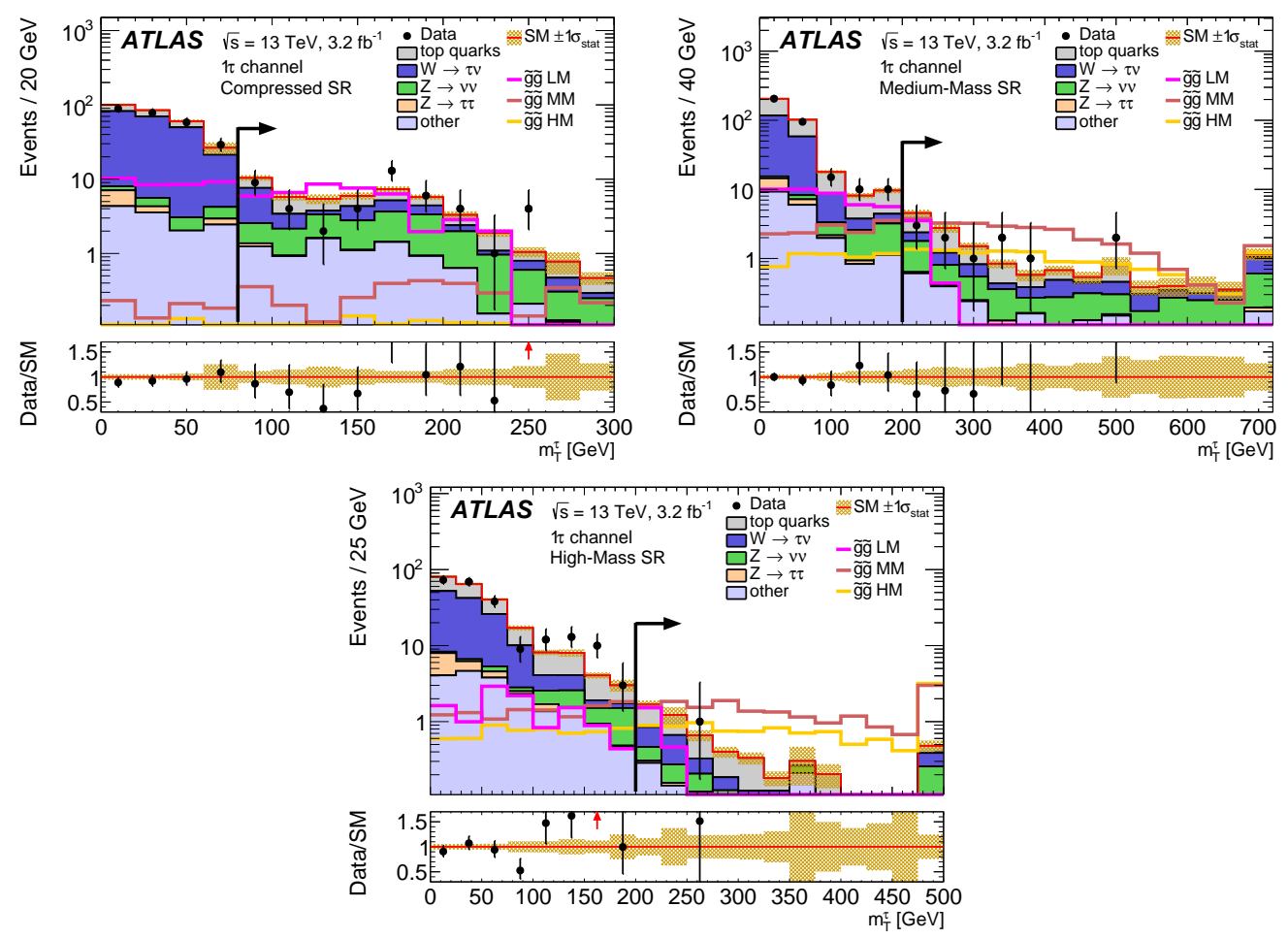

Figure 15: Distribution of $m_{\mathrm{T}}^{\tau}$ is the signal regions of the single $\tau$ channel of the ATLAS $\tau$ lepton search. From left to right, the signal regions target low, medium, and high masses of the initially produced SUSY particles. [11].

the large improvement in sensitivity gained by the increase in center-of-mass energy from $8 \mathrm{TeV}$ to $13 \mathrm{TeV}$ is evident. For $m_{\tilde{\chi}_{1}^{0}} \approx 100 \mathrm{GeV}, m_{\tilde{g}}$ below $1.57 \mathrm{TeV}$ is excluded. The limit on $m_{\tilde{\chi}_{1}^{0}}$ reaches $700 \mathrm{GeV}$ for $m_{\tilde{g}}$ between 0.8 and $1.5 \mathrm{TeV}$. The interpretation of the results in the context of the GSMB model is shown on the right side of Figure 16. Again, there is large improvement due to the increase in centre-of-mass energy. For all considered values of $\tan \beta, \Lambda<92 \mathrm{TeV}$ is excluded. This corresponds to a limit on $m_{\tilde{g}}$ of $2 \mathrm{TeV}$. For high values of $\tan \beta$, the results exclude $\Lambda<107 \mathrm{TeV}$ and $m_{\tilde{g}}<2.3 \mathrm{TeV}$.

\section{Searches with photons}

Final states with photons are common in GMSB models. In the models studies by ATLAS and CMS $[13,12]$, the gravitino is the stable LSP and the $\tilde{\chi}_{1}^{0}$ is assumed to be the NLSP. The decays of the $\tilde{\chi}_{1}^{0}$ depend on their gaugino content. Especially, a high admixture of the bino, as is common in GMSB, leads to a high branching fraction for the decay $\tilde{\chi}_{1}^{0} \rightarrow \tilde{G} \gamma$. Both ATLAS and CMS consider a model of $\tilde{g}$ pair production, where the $\tilde{g}$ decays according to $\tilde{g} \rightarrow q \bar{q} \tilde{\chi}_{1}^{0}$. In the CMS search, the $\tilde{\chi}_{1}^{0}$ decays to $\tilde{G} \gamma$ with $100 \%$ probability. ATLAS assumes a more realistic branching fraction, which is $100 \%$ for $m_{\tilde{\chi}_{1}^{0}} \rightarrow 0$ and approaches $\cos ^{2} \theta_{\mathrm{W}}$ for $m_{\tilde{\chi}_{1}^{0}} \gg m_{\mathrm{Z}}$. The diagram for this model is shown in Figure 17.

The resulting final state features two photons, several jets, and $E_{\mathrm{T}}^{\mathrm{miss}}$. Several types of SM processes contribute to this final state: QCD processes with two real photons or misidentified jets, 

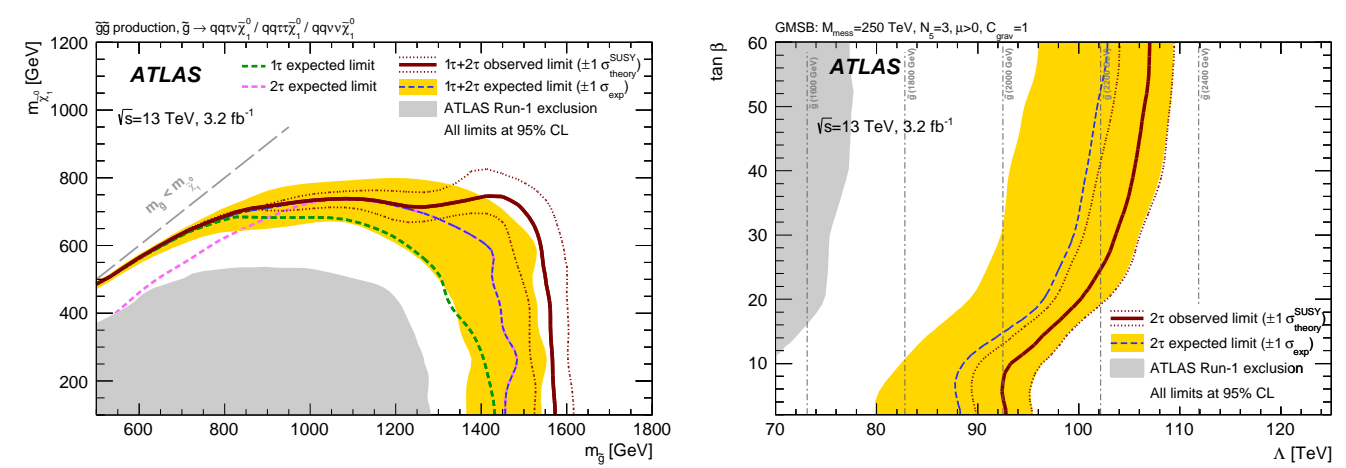

Figure 16: Left: Exclusion limits in the simplified model featuring $\tilde{g}$ pair production in the $m_{\tilde{g}}-m_{\tilde{\chi}_{1}^{0}}$-plane in the ATLAS $\tau$ lepton search [11]. Right: Exclusion limits in the $\Lambda-\tan \beta$-plane for the GMSB model in the ATLAS $\tau$ lepton search [11].

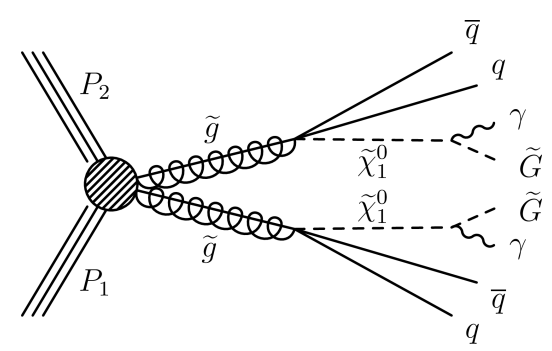

Figure 17: Diagram of the model searched for in the ATLAS and CMS diphoton searches [12].

vector boson or top-pair production where electrons are misidentified as photons, and vector boson production in association with two prompt photons $(\mathrm{W} \gamma \gamma, \mathrm{Z} \gamma \gamma)$. These backgrounds are estimated mostly from data in both the ATLAS and CMS searches.

In the CMS search, events are selected requiring two photons with $p_{\mathrm{T}}>40 \mathrm{GeV}, E_{\mathrm{T}}^{\text {miss }}>$ $100 \mathrm{GeV}$ and diphoton invariant mass $m_{\gamma \gamma}>105 \mathrm{GeV}$. The backgrounds contributing to this selection are mostly QCD processes, where $E_{\mathrm{T}}^{\text {miss }}$ is caused by jet mismeasurement, and $\mathrm{W} \gamma$ production, where the W decays to $e v$ and the electron is misidentified as a photon. The former background is estimated from a dielectron control sample, while the estimation for the latter is derived from an $e \gamma$ control sample. The left side of Figure 18 shows the final $E_{\mathrm{T}}^{\text {miss }}$ distribution in the signal region. There is good agreement between the observed data and the background estimates. This result is interpreted in the model shown in Figure 17. The resulting exclusion limits are shown on the right side of Figure 18. An lower bound on $m_{\tilde{g}}$ of up to $1.65 \mathrm{TeV}$ is set, improving the limit derived at $\sqrt{s}=8 \mathrm{TeV}$ by $300 \mathrm{GeV}$.

The ATLAS diphoton search has been optimized for maximum sensitivity at high $m_{\tilde{g}}$, resulting in comparably strong requirements on the events. The signal selection requires two photons with $p_{\mathrm{T}}>75 \mathrm{GeV}, m_{\mathrm{eff}}>1500 \mathrm{GeV}, E_{\mathrm{T}}^{\text {miss }}>175 \mathrm{GeV}$, and $\Delta \Phi_{\min }\left(\right.$ jet, $\left.E_{\mathrm{T}}^{\text {miss }}\right)>0.5$. The background from QCD processes with two real photons is estimated from simulation, which has been reweighted to match the data in the $E_{\mathrm{T}}^{\text {miss }}$ and $N_{\text {jet }}$ distributions. QCD processes with misidentified photons are estimated from non-isolated photons. As in the CMS search, an $e \gamma$ control sample is used to estimate backgrounds where an electron is misidentified as a photon. In this search $\mathrm{W} \gamma \gamma$ 

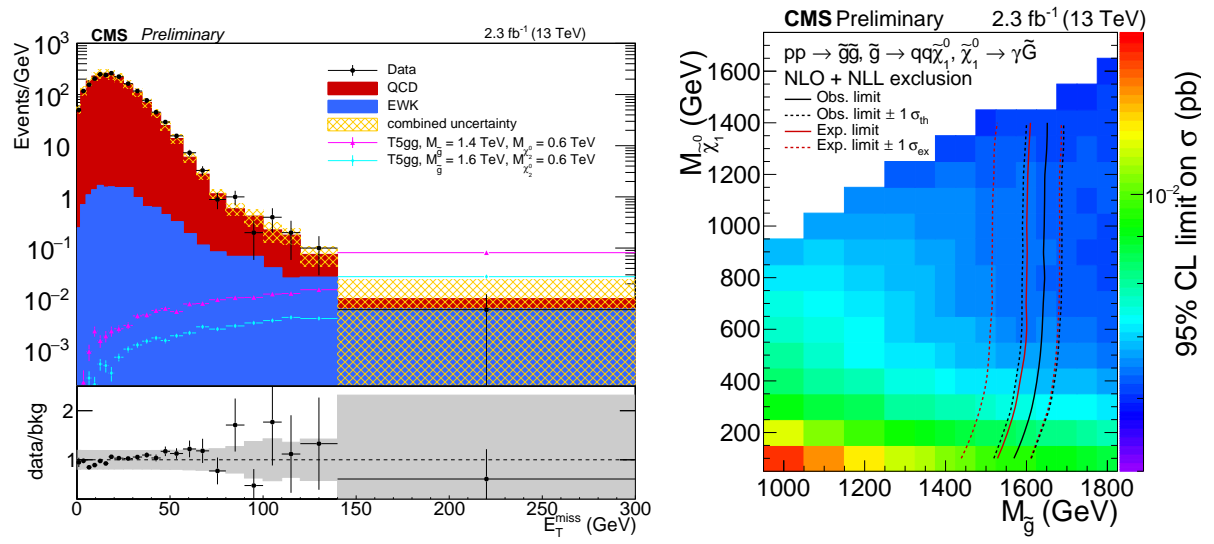

Figure 18: Left: Final $E_{\mathrm{T}}^{\text {miss }}$ distribution in the CMS diphoton search [12]. Right: Exclusion limits in the CMS diphoton search [12].

is the dominant background. It is estimated from simulation, which has been normalized to data in a control region. The subdominant $\mathrm{Z} \gamma \gamma$ background is estimated directly from simulation. The distributions of $E_{\mathrm{T}}^{\mathrm{miss}}$ and $m_{\mathrm{eff}}$ before applying the signal region requirements on both observables, are shown in Figure 19 to illustrate the good description of the observed data by the background estimates.
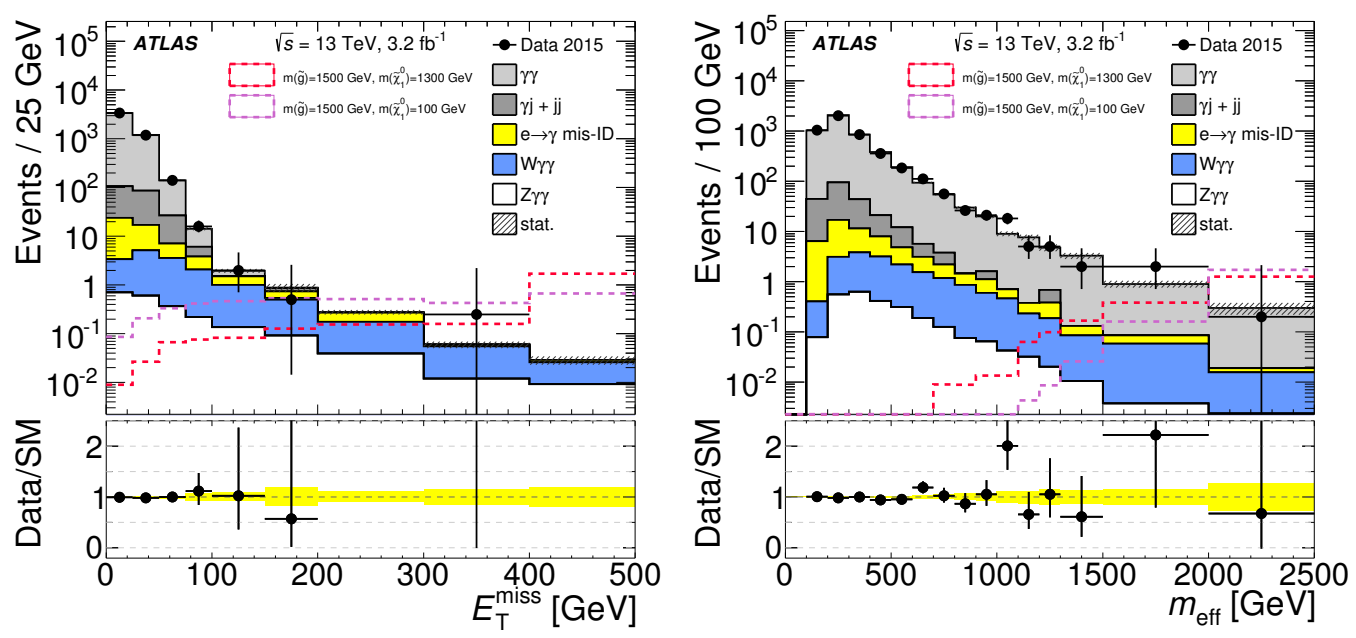

Figure 19: Left: Distribution of $E_{\mathrm{T}}^{\text {miss }}$ in the ATLAS diphoton search before application of the $m_{\mathrm{eff}}$ requirement [13]. Right: Distribution of $m_{\mathrm{eff}}$ in the ATLAS diphoton search before application of the $E_{\mathrm{T}}^{\text {miss }}$ requirement [13].

In the signal region, $0.27_{-0.10}^{+0.22}$ events are expected and zero events are observed. Figure 20 shows the resulting exclusion limits on the signal model described above. Lower bounds on $m_{\tilde{g}}$ ranging from $1650-1750 \mathrm{GeV}$ are set.

\section{Summary}

An extensive search program for Supersymmetry is performed at the LHC by the ATLAS 


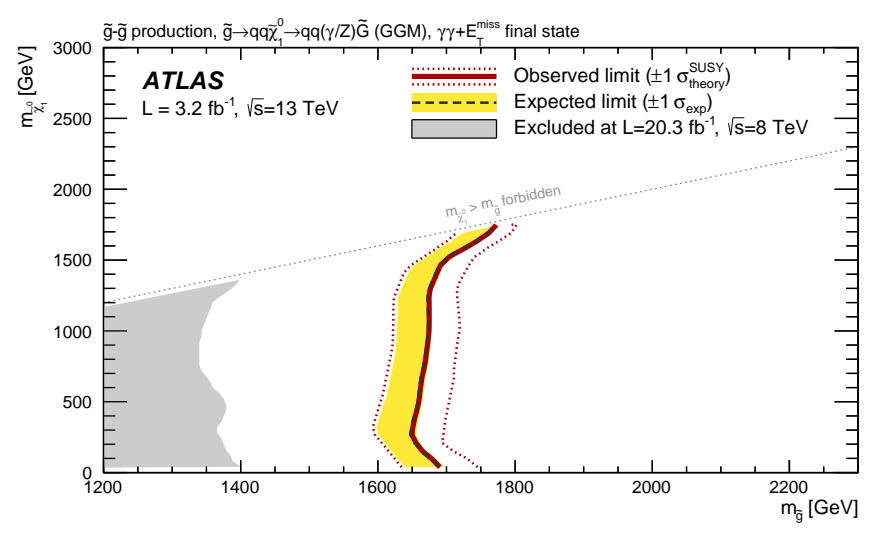

Figure 20: Resulting exclusion limits in the ATLAS diphoton search [13].

and CMS collaborations. Final states with leptons and photons offer access to unique parameter spaces and different SUSY breaking mechanisms, making them an integral part of this effort. The datasets recorded at $\sqrt{s}=13 \mathrm{TeV}$ in 2015 greatly increase the sensitivity of most SUSY searches and the results were highly anticipated. The analysis of the datasets recorded at $\sqrt{s}=8 \mathrm{TeV}$ had left us with two interesting excesses in the opposite-sign dilepton searches, which made revisiting this final state especially interesting. The excess observed by CMS for dilepton invariant masses below the $\mathrm{Z}$ boson peak at $\sqrt{s}=8 \mathrm{TeV}$ was not confirmed in the analysis of the $\sqrt{s}=13 \mathrm{TeV}$ data. However, ATLAS did see again a small excess of $2.2 \sigma$ local significance in their search for SUSY models producing Z bosons in the decay chains. This excess was not confirmed by CMS in their analysis of the $\sqrt{s}=13 \mathrm{TeV}$ dataset. Therefore, this final state promises to stay interesting as more data is collected during the 2016 LHC run. In a variety of other searches, good agreement between the observed data and background estimates is observed. This translates into limits on the gluino mass of 1.1-1.3 TeV in a multitude of simplified models. They increase to over $2 \mathrm{TeV}$ in models with gauge mediated SUSY breaking. Lower limits on the bottom-squark mass in the range of $540-680 \mathrm{GeV}$ are set, depending on the specific model and search. A much larger data sample at $\sqrt{s}=13 \mathrm{TeV}$ has already been recorded during the 2016 LHC run, so a further increase in sensitivity is expected and these final states remain a topic of continuing interest.

\section{References}

[1] L. Evans and P. Bryant, "LHC Machine", JINST 3 (2008) S08001, doi:10.1088/1748-0221/3/08/S08001.

[2] ATLAS Collaboration, "The ATLAS Experiment at the CERN Large Hadron Collider", JINST 3 (2008) S08003, doi:10.1088/1748-0221/3/08/S08003.

[3] CMS Collaboration, "The CMS Experiment at the CERN Large Hadron Collider", JINST 3 (2008) S08004, doi:10.1088/1748-0221/3/08/S08004.

[4] CMS Collaboration, "Search for physics beyond the standard model in events with two leptons, jets, and missing transverse momentum in pp collisions at $\sqrt{s}=8 \mathrm{TeV}$ ", JHEP 1504 (2015) 124, doi:10.1007/JHEP04(2015)124, arXiv:1502.06031. 
[5] ATLAS Collaboration, "Search for supersymmetry in events containing a same-flavour opposite-sign dilepton pair, jets, and large missing transverse momentum in $\sqrt{s}=8 \mathrm{TeV}$ pp collisions with the ATLAS detector", Eur. Phys. J C75 (2015) 318, doi:10.1140/epjc/s10052-015-3661-9, arXiv:1503.03290.

[6] CMS Collaboration, "Search for new physics in final states with two opposite-sign, same-flavor leptons, jets, and missing transverse momentum in pp collisions at $\sqrt{s}=13 \mathrm{TeV}$ ", arXiv:1607.00915, Submitted to JHEP.

[7] ATLAS Collaboration,"A search for Supersymmetry in events containing a leptonically decaying $Z$ boson, jets and missing transverse momentum in $\sqrt{s}=13 \mathrm{TeV} p p$ collisions with the ATLAS detector", ATLAS-CONF-2015-082, http: / / cds. cern. ch/record/2114854.

[8] ATLAS Collaboration, "Search for supersymmetry at $\sqrt{s}=13 \mathrm{TeV}$ in final states with jets and two same-sign leptons or three leptons with the ATLAS detector", Eur. Phys. J C76 (2016) 259, doi:10.1140/epjc/s10052-016-4095-8, arXiv:1602.09058.

[9] CMS Collaboration, "Search for new physics in same-sign dilepton events in proton-proton collisions at $\sqrt{s}=13 \mathrm{TeV} "$, Eur. Phys. J C76 (2016) 439, doi:10.1140/epjc/s10052-016-4261-z, arxiv:1605.03171.

[10] CMS Collaboration, "Search for SUSY with multileptons in $13 \mathrm{TeV}$ data ", CMS-PAS-SUS-16-003, https://cds.cern.ch/record/2140637.

[11] ATLAS Collaboration, "Search for squarks and gluinos in events with hadronically decaying tau leptons, jets and missing transverse momentum in proton-proton collisions at $\sqrt{s}=13 \mathrm{TeV}$ recorded with the ATLAS detector", (2016) arXiv:1607.05979, Submitted to EPJC.

[12] CMS Collaboration, "Search for supersymmetry in the multijet and missing transverse momentum final state in pp collisions at 13 TeV", Phys. Lett. B 758 (2016) 152, doi:10.1016/j.physletb.2016.05.002, arxiv:1602.06581.

[13] ATLAS Collaboration, "Search for supersymmetry in a final state containing two photons and missing transverse momentum in $\sqrt{s}=13 \mathrm{TeV} p p$ collisions at the LHC using the ATLAS detector", (2016) arXiv:1606.09150, Submitted to EPJC. 